\title{
A New Adaptability Assessment Algorithm for Camouflage Screen using Super-efficiency DEA
}

\author{
Ling Li, Zhiyao Yue, Heng Liu* \\ National Key Laboratory on Environmental Electromagnetic Effects and Electro-Optic Engineering \\ College of Field Engineering, PLA University of Science and Technology \\ Nanjing, China \\ liuhenglgdx@163.com
}

\begin{abstract}
In view of disadvantages of conventional approaches such as insufficient ranking, disunity of index dimensions as well as too subjective weights in adaptability assessment of camouflage screen, we proposed a new super-efficiency DEA algorithm to overcome them. Traditional DEA algorithm often runs into several DEA efficient decision making unit simultaneously and the efficiency score of a DMU is denoted by a unity, which leads to unsatisfactory differentiation of all units. On the basis of CCR model, we removed the evaluated DMU itself and compare it with linear combination of other DMUs to formulate super-efficiency DEA. It can rank all the DMUs completely, thus it has better distinguish ability. Moreover, DEA is not influenced by index dimensions, and its weights had inner objectivity. The actual example shows that the super-efficiency DEA can realize effective adaptability assessment of multi-band camouflage screen and avoid the influence of dimensions and weights. The algorithm can provide us a practical model for camouflage design and evaluation.
\end{abstract}

Keywords-camouflage screen; adaptability assessment; superefficiency DEA; sufficient ranking; weight

\section{INTRODUCTION}

Camouflage screen adaptability assessment has become an interesting subject of many scholars in recent years due to its vital role in camouflage technology. It is a multiple criteria decision making essentially, showing solicitude for suitable index set and effective evaluating measures. Multi-band camouflage screen design involves visible light, near infrared, far infrared, microwave et al., which is a multiple criteria decision making essentially. For a long time, scheme selection decision of multi-band camouflage screen design goes to be rough and qualitative. When it faces multiple criteria evaluation, there are few practical models. Meanwhile, these conventional models such as fuzzy comprehensive evaluation and grey analysis cannot realize sufficient assessment and objective weights.

Data envelopment analysis (DEA), originated by Charnes, Cooper, and Rhodes, is a non-parametric approach for evaluating the relative efficiency of homogeneous decision making units (DMUs), which use similar inputs to produce similar outputs [1]. DEA assesses the efficiency of each DMU relative to an estimated production possibility frontier formed by all DMUs. The advantage of DEA approach is that it requires no assumption on the appearance of the frontier surface as well as it makes no hypothesis concerning the internal operations of a DMU.

In DEA model, the best DMUs are called DEA efficient and the efficiency score of a DMU is denoted by a unity, which leads to unsatisfactory differentiation of all the DMUs. In order to solve this problem, ranking DEA efficient DMUs has become an interesting topic of many scholars and a number of models (called super-efficiency models) were explored to rank DEA efficient DMU. Among these models, one can mention Andersen and Petersen's [2] super-efficiency model and the slack-based measure (SBM) introduced by Tone [3]. In recent years, the super-efficiency model for SBM has been developed by many researchers for the purpose of ranking SBM efficient DMUs [4] [5] [6]. A.A. Noura, et al. [7] developed the ranking method according to the weights and the desirability of inputs and outputs. In parallel, a number of literatures have studied the problem of super-efficiency model's infeasibility (see, e.g., [8] [9] [10]).

\section{ADAPTABILTY ASSESSMENT INDEX SYSTEM FOR CAMOUFLAGE SCREEN}

There are many factors that influence the performance of multi-band camouflage screen. We take index system into consideration from the following principles: (1) Comprehensiveness and simplicity must be concerned about simultaneously to cover the most important influence indexes of camouflage screen. (2) Irrelevance should be thought over between every two indexes of input and output. (3) Availability of index data can be another crucial factor we must take into consideration.

In view of DEA structure, input and output index have their normal requirements: input is inversely proportional to the DEA efficiency; output is proportional to the DEA efficiency. Thus, we classify the indexes into two categories of cost and benefit index, which can be described as input and output respectively. According to practical application of multi-band camouflage screen, we extract seven representative indexes to set up index system. We can classify them by two categories: we deal with radiation temperature $\left(x_{1}\right)$, infrared emissivity $\left(x_{2}\right)$ and RCS $\left(x_{3}\right)$ as inputs, and handle speckle reflectivity $\left(y_{1}\right)$, speckle size $\left(y_{2}\right)$, speckle color $\mathrm{X}$ value $\left(y_{3}\right)$ and speckle color 


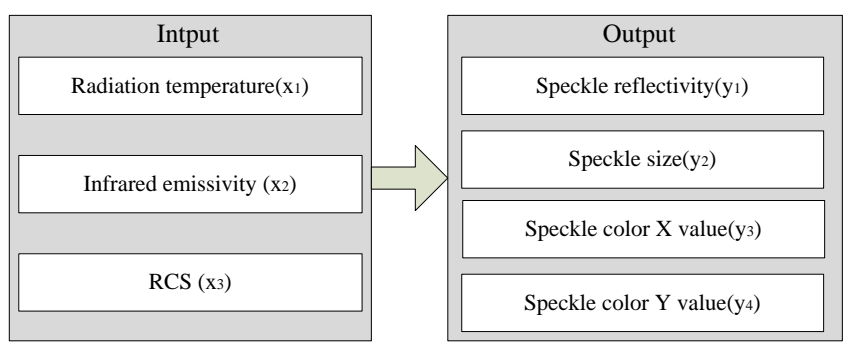

Figure 1. Adaptability assessment index system for camouflage screen

\section{SUPER-EFFICIENCY DEA ALGORITHM}

DEA is a widely used mathematical programming approach for comparing the multiple inputs and outputs of a set of homogenous DMUs by measuring their relative efficiency. Suppose that there are $n$ DMUs to be measured where $j$ th DMU is defined as $D M U_{j}, j=1,2, \ldots, n$, consuming $m$ inputs, $x_{i j}(i=$ $1,2, \ldots, m)$, producing $s$ outputs, $y_{r j}(r=1,2, \ldots, s)$, which can be described as Fig .2.

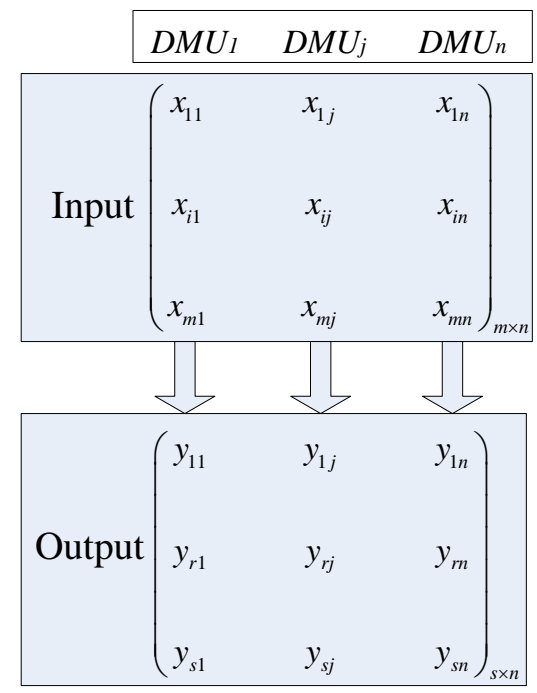

Figure 2. Input and output structure of DEA model

$$
\begin{array}{ll}
\max & q_{0}=\sum_{r=1}^{s} \mu_{r} y_{r 0} \\
\text { s.t. } & \sum_{i=1}^{m} \omega_{i} x_{i 0}=1 \\
& \sum_{i=1}^{m} \omega_{i} x_{i j}-\sum_{r=1}^{s} \mu_{r} y_{r j} \geq 0 \\
& j=1,2, \mathrm{~L} n \\
& \omega_{i}, \mu_{r} \geq 0, \quad \forall i, r
\end{array}
$$

Suppose that there are $n$ DMUs to be evaluated where each $D M U_{j}, j=1,2, \ldots, n$, consumes $m$ inputs, $x_{i j}(i=1,2, \ldots, m)$, producing $s$ outputs, $y_{r j}(r=1,2, \ldots, s)$. The CCR model for evaluating the technical input-efficiency of $j_{0}$ th DMU $\left(D M U_{0}\right)$ under constant returns to scale (CRS) is represented as Eq. 1.

The dual model for assessing the input-orientated efficiency of $D M U_{0}$ under CRS with non-Archimedean infinitesimal is formulated as Eq. 2. Where $\hat{e}=(1,1, \ldots$, $1)^{T} \in E^{m}$ and $e=(1,1, \ldots, 1)^{T} \in E^{s}$ are unit vectors, and $\varepsilon$ $(\varepsilon>0)$ is a non-Archimedean infinitesimal. $S^{+}$and $S^{-}$are slacks, which reflect non-radial improvement between one DMU and its optimal condition. Slack based measure can provide us more management information about improvement. We construct the model under CRS, offering us overall efficiency, which includes technical efficiency under variable returns to scale (VRS) and scale effect.

$$
\begin{array}{cl}
\min & {\left[\theta-\varepsilon\left(\hat{e}^{T} S^{-}+e^{T} S^{+}\right)\right]} \\
\text {s.t. } & \sum_{j=1}^{n} X_{j} \lambda_{j}+S^{-}=\theta X_{0} \\
& \sum_{j=1}^{n} Y_{j} \lambda_{j}-S^{+}=Y_{0} \\
& \lambda_{j} \geq 0, \quad j=1,2, \mathrm{~L}, n \\
& S^{-} \geq 0, S^{+} \geq 0
\end{array} .
$$

Definition 1. On the basis of the Eq. 2, we can classify the DMUs into one of the following two categories:

(1) The efficient category: all the DMUs that are always DEA efficient. That is, the efficiency is equal to unity: $C=\left\{D M U_{j} \mid \theta_{j}=1, j=1,2, \mathrm{~L}, n\right\}$.

(2) The inefficient category: all the DMUs that are always DEA inefficient. That is, the efficiency is smaller than unity: $C^{-}=\left\{D M U_{j} \mid \theta_{j}<1, j=1,2, \mathrm{~L}, n\right\}$.

The efficiencies of efficient DMUs in Eq. 1 are equal to unity simultaneously, which leads to unsatisfactory discrimination. Anderson and Petersen have suggested the super-efficiency model to arrive at a ranking of all efficient DMUs, which can be applied to differentiate efficient DMUs in DEA. As shown in Fig .3, $C$ is in the PPS $A B C D E$, which denotes that the efficiency of $C$ is equal to unity. When we 
eliminate $C$ from the solution set and compare it with linear combination of other points, PPS changes to be $A B D E$. The extension line of $O C$ converges with $B D$ in $C^{\prime}$, showing the super-efficiency of $\mathrm{C}$ is changed to $O C^{\prime} / O C$, which is greater than unity. Thus, the obtained super-efficiencies of efficient DMUs are greater than or equal to unity, leading to complete ranking of all the DMUs.

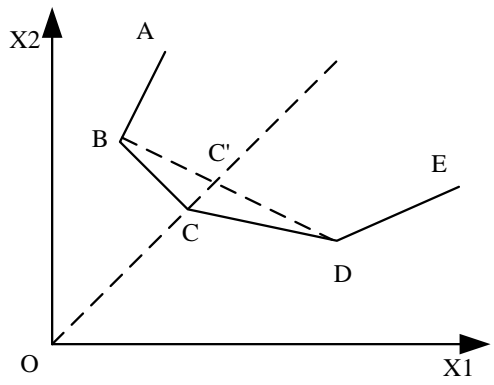

Figure 3. Illustrative diagram of SE-DEA

Conventional DEA can be extended into SE-DEA, which is proposed as Eq. 3.

$$
\begin{array}{ll}
\min & {\left[\alpha-\varepsilon\left(\hat{e}^{T} S^{-}+e^{T} S^{+}\right)\right]} \\
\text {s.t. } & \sum_{j=1, j \neq 0}^{n} X_{j} \lambda_{j}+S^{-}=\alpha X_{0} \\
& \sum_{j=1, j \neq 0}^{n} Y_{j} \lambda_{j}-S^{+}=Y_{0} \\
& \lambda_{j} \geq 0, j=1,2, \mathrm{~L}, n, j \neq 0 \\
& S^{-} \geq 0, S^{+} \geq 0
\end{array} .
$$

Definition 2. On the basis of the Eq. 3, we can classify the DMUs into one of the following two categories:

(1) The efficient category: all the DMUs that are always super-efficient. That is, the efficiency is more than or equal to unity: $E=\left\{D M U_{j} \mid \alpha_{j} \geq 1, j=1,2, \mathrm{~L}, n\right\}$.

(2) The inefficient category: all the DMUs that are always super-inefficient. That is, the efficiency is smaller than unity: $E^{-}=\left\{D M U_{j} \mid \alpha_{j}<1, j=1,2, \mathrm{~L}, n\right\}$.

\section{NUMERICAL EXAMPLE}

In this section, a numerical example is presented to illustrate the application procedure of camouflage screen adaptability assessment using the approach suggested above. To this end, ten DMUs with three inputs and four outputs are considered, which can be listed in TAB. I.

We run conventional DEA and super-efficiency DEA respectively. The assessment results can be obtained as shown in TAB. II. We know $\lambda_{j} \geq 0$ according to Eq. 3, which requires no subjective setting. The efficiency of $D M U_{7}$ and $D M U_{9}$ are both equal to a unity, which leads to unsatisfactory discrimination. From super-efficiency DEA, we can obtain that $\alpha_{7}=1.13$ and $\alpha_{9}=1.27$, providing us sufficient ranking of all DMUs. We can conclude that $D M U_{9}$ performs best, and $D M U_{10}$ displays worst.

From TAB. II we can conclude that inefficient DMUs are also super-inefficient. This is because the inefficient DMUs are not on the production frontier, which is also means envelopment surface. Thus, super-efficiency running cannot vary the efficient status. We can gain the ranking sequence of camouflage screen adaptability assessment as following: $D M U_{9}>D M U_{7}>D M U_{8}>D M U_{1}>D M U_{2}>D M U_{3}>D M U_{4}>D M U_{5}>$ $D M U_{6}>D M U_{10}$.

TABLE I. InPUT AND OUtPut DATA OF Ten CAMOUFLAGE SCREen

\begin{tabular}{|l|c|c|c|c|c|c|c|c|c|c|}
\hline & $\mathbf{D M U}_{\mathbf{1}}$ & $\mathbf{D M U}_{\mathbf{2}}$ & $\mathbf{D M U}_{\mathbf{3}}$ & $\mathbf{D M U}_{\mathbf{4}}$ & $\mathbf{D M U}_{\mathbf{5}}$ & $\mathbf{D M U}_{\mathbf{6}}$ & $\mathbf{D M U}_{\mathbf{7}}$ & $\mathbf{D M U}_{\mathbf{8}}$ & $\mathbf{D M U}_{\mathbf{9}}$ & $\mathbf{D M U}_{\mathbf{1 0}}$ \\
\hline$x_{1}$ & 21.6 & 18.0 & 22.95 & 24.3 & 22.5 & 19.8 & 18.0 & 25.2 & 18.9 & 26.1 \\
\hline$x_{2}$ & 0.81 & 0.837 & 0.765 & 0.801 & 0.846 & 0.837 & 0.819 & 0.792 & 0.702 & 0.855 \\
\hline$x_{3}$ & 0.522 & 0.675 & 0.801 & 0.693 & 0.621 & 0.9 & 0.612 & 0.801 & 0.504 & 0.819 \\
\hline$y_{1}$ & 0.171 & 0.18 & 0.207 & 0.153 & 0.234 & 0.18 & 0.252 & 0.216 & 0.243 & 0.189 \\
\hline$y_{2}$ & 2.25 & 2.07 & 1.89 & 2.16 & 1.98 & 1.8 & 2.43 & 2.52 & 2.25 & 1.89 \\
\hline$y_{3}$ & 0.333 & 0.306 & 0.135 & 0.342 & 0.252 & 0.225 & 0.315 & 0.297 & 0.351 & 0.144 \\
\hline$y_{4}$ & 0.585 & 0.594 & 0.567 & 0.423 & 0.54 & 0.522 & 0.639 & 0.432 & 0.603 & 0.396 \\
\hline
\end{tabular}

TABLE II. AdAPtABILITy AsSESSMENT Results of CAMOUFLAGE SCREEN APPLYING IMPRoved DEA Algorithm

\begin{tabular}{|c|c|c|c|c|c|c|c|c|c|c|}
\hline & $\mathbf{D M U}_{\mathbf{1}}$ & $\mathbf{D M U}_{\mathbf{2}}$ & $\mathbf{D M U}_{\mathbf{3}}$ & $\mathbf{D M U}_{\mathbf{4}}$ & $\mathbf{D M U}_{\mathbf{5}}$ & $\mathbf{D M U}_{\mathbf{6}}$ & $\mathbf{D M U}_{\mathbf{7}}$ & $\mathbf{D M U}_{\mathbf{8}}$ & $\mathbf{D M U}_{\mathbf{9}}$ & $\mathbf{D M U}_{\mathbf{1 0}}$ \\
\hline$\theta$ & 0.97 & 0.96 & 0.86 & 0.85 & 0.81 & 0.77 & $\mathbf{1}$ & 0.99 & $\mathbf{1}$ & 0.69 \\
\hline Classification of CCR & $C$ & $C$ & $C$ & $C$ & $C$ & $C$ & $C$ & $C$ & $C$ & $C$ \\
\hline$\alpha$ & 0.97 & 0.96 & 0.86 & 0.85 & 0.81 & 0.77 & $\mathbf{1 . 1 3}$ & 0.99 & $\mathbf{1 . 2 7}$ & 0.69 \\
\hline Classification of SE-DEA & $E$ & $E$ & $E$ & $E$ & $E$ & $E$ & $E$ & $E$ & $E$ & $E$ \\
\hline Ranking & 4 & 5 & 6 & 7 & 8 & 9 & 2 & 3 & 1 & 10 \\
\hline
\end{tabular}




\section{CONCLUSIONS}

This paper put forward a new algorithm for camouflage screen adaptability assessment based on super-efficiency DEA with non-Archimedean infinitesimal. We extracted radiation temperature, infrared emissivity, RCS, speckle reflectivity, speckle size and speckle color values (X and Y) of multi-band camouflage screen to construct index system. On the basis of CCR model, we removed the evaluated DMU itself and compare it with linear combination of other DMUs to formulate super-efficiency DEA. It can rank all the DMUs completely and has better distinguish ability. Simultaneously, DEA is not influenced by index dimensions, and its weights had inner objectivity. An actual example shows that the superefficiency DEA can realize sufficient ranking of camouflage screen schemes and avoid the influence of dimensions and weights. Further research could extend the applied model to include fuzzy data or network structure.

\section{ACKNOWLEDGMENT}

This work is supported by Foundation of National Key Laboratory on Environmental Electromagnetic Effects and Electro-Optic Engineering (Grand No. FD2015008).

\section{REFERENCES}

[1] A. Charnes, W.W. Cooper, and E. Rhodes, "Measuring the efficiency of decision making units," European Journal of Operational Research, 2 (1978), pp. 429-444.
[2] P. Andersen, and N.C. Petersen, "A procedure for ranking efficient units in data envelopment analysis," Management Science, 39, 10(1993), pp. 1261-1264.

[3] K. Tone, "A slacks-based measure of efficiency in data envelopment analysis," European Journal of Operational Research, 130, 3(2001), pp. 498-509.

[4] Hsin-Hsiung Fang, Hsuan-Shih Lee, Shiuh-Nan Hwang, and Cheng-Chi Chung, "A slacks-based measure of super-efficiency in data envelopment analysis:An alternative approach," Omega-International Journal of Management Science, 41, 4(2013), pp. 731-734.

[5] Hsiao Bo, Chern Ching-Chin, Chiu Yung-Ho, and Chiu Ching-Ren, "Using fuzzy super-efficiency slack-based measure data envelopment analysis to evaluate Taiwan's commercial bank efficiency," Expert Systems with Applications, 38, 8(2011), pp. 9147-9156.

[6] Chiu Yung-Ho, Chen Yu-Chuan, and Bai Xue-Jie, "Efficiency and risk in Taiwan banking: SBM super-DEA estimation," Applied Economics, 43, 5(2011), pp. 587-602.

[7] A.A. Noura, F. Hosseinzadeh Lotfi, G.R. Jahanshahloo, and S. Fanati Rashidi, "Super-efficiency in DEA by effectiveness of each unit in society," Applied Mathematics Letters, 24, 5(2011), pp. 623-626.

[8] Esmaeilzadeh A., and Hadi-Vencheh A, "A super-efficiency model for measuring aggregative efficiency of multi-period production systems,' Measurement, 46, 10(2013), pp. 3988-3993.

[9] Amirteimoori Alireza, and Kordrostami Sohrab, "A distance-based measure of super efficiency in data envelopment analysis: an application to gas companies," Journal of Global Optimization, 54, 1(2012), pp. 117-128.

[10] Chen Jin-Xiao, Deng Mingrong, and Gingras Sylvain, “A modified super-efficiency measure based on simultaneous input-output projection in data envelopment analysis," Computers \& Operations Research, 38 , 2(2011), pp. 496-504 